\title{
WIBRAGEC

\section{GESTÃO DA OPERAÇÃO E MANUTENÇÃO INTEGRADA AO BUILDING INFORMATION MODELING: ANÁLISE CIENTOMÉTRICA ${ }^{1}$}

\author{
MORAIS, Gabriela Alves Tenório de (1); PALHA, Rachel Perez (2) \\ (1) Universidade Federal de Pernambuco, gabriela_morais@outlook.com \\ (2) Universidade Federal de Pernambuco, rachel.palha@ufpe.br
}

\begin{abstract}
RESUMO
A Operação e Manutenção $(O \& M)$ de instalações habilitadas para o Building Information Modeling (BIM) se apresenta como um emergente campo de pesquisa. No entanto, a maioria das pesquisas sobre BIM estão relacionadas às etapas de planejamento, projeto e execução. As aplicações do BIM para O\&M, fase mais longa e com maior contribuição nos custos do ciclo de vida das construções, ainda são incipientes. $O$ objetivo desta pesquisa é realizar uma análise cientométrica e crítica da literatura científica acerca da O\&M de instalações habilitadas para o BIM. Os dados analisados se baseiam em 1359 trabalhos publicados, desde o início da indexação de publicações (1945-2021), na base de dados Web of Science com auxílio das ferramentas VOSviewer e CiteSpace. A rede de países, obtida por meio da ferramenta VOSviewer, demonstrou que o Brasil ainda precisa ampliar o número de publicações neste campo e ampliar suas redes de colaboração. Através do CiteSpace foi possível apontar linhas de pesquisa e identificar que tópicos relacionados aos sistemas de informação geográfica e edificações escolares têm sido trabalhados mais recentemente. Esta pesquisa contribui para ampliação das fronteiras do conhecimento ao analisar o estado da arte e identificar tendências de pesquisa.
\end{abstract}

Palavras-chave: Modelagem da informação da construção, O\&M, Análise cientométrica.

\begin{abstract}
The Operation and Maintenance $(O \& M)$ of facilities enabled for Building Information Modeling (BIM) presents itself as an emerging field of research. However, most research on BIM is related to the planning, design, and execution stages. The applications of BIM for $O \& M$, which is the most extended phase and has the most significant contribution to the life cycle costs of buildings, are still incipient. This research aims to carry out a scientometric and critical analysis of the scientific literature about the O\&M of facilities enabled for BIM. The analyzed data are based on 1359 papers published since the beginning of indexing publications (1945-2021) in the Web of Science database with the VOSviewer and CiteSpace tools' aid. The network of countries, obtained through the VOSviewer tool, demonstrated that Brazil still needs to increase the number of publications in this field and expand its collaboration networks. Through CiteSpace, it was possible to point outlines of research; it was identified that topics related to geographic information systems and school buildings had been worked on more recently. This research contributes to expanding the frontiers of knowledge by analyzing state of the art and identifying research trends.
\end{abstract}

Keywords: building information modeling, $O \& M$, scientometric analysis.

\section{INTRODUÇÃO}

A Modelagem da Informação da Construção (Building Information Modeling - BIM), é uma das maiores inovações da indústria da Arquitetura, Engenharia e Construção (AEC)

\footnotetext{
${ }^{1}$ MORAIS, G. A. T. de; PALHA, R. P. Gestão da operação e manutenção integrada ao Building Information Modeling: análise cientométrica e revisão crítica. In: SIMPÓSIO BRASILEIRO DE GESTÃO E ECONOMIA DA CONSTRUÇÃO, 12., 2021, Maceió. Anais[...] Porto Alegre: ANTAC, 2021. p.1-8. Disponível em: https://eventos.antac.org.br/index.php/sibragec/article/view/490. Acesso em: 2 out. 2021.
} 
(OKAKPU et al., 2020). Porém, ainda que esteja em ascensão (FITZ; SALEEB, 2019), ganhou popularidade somente na última década (FAROOQ; SHARMA; SREERAMA KUMAR, 2017). Neste âmbito, a revisão das ferramentas BIM aplicadas nas construções da China apresentada por Li et al. (2018) demonstrou que as aplicações destas ferramentas para as atividades de Operação e Manutenção (O\&M) ainda são incipientes. Gao e Pishad-Bozorgi (2019) afirmam que os estudos que tratam do uso do BIM para a O\&M de construções ainda estão em estágios iniciais. Na mesma direção, Muller et al. (2019) apontam que as pesquisas acadêmicas que tratam do uso do BIM ao longo do ciclo de vida das construções tendem a dedicar menor atenção às atividades de manutenção e demolição. Para Dong a, O'Neill e Li (2014), as limitações do uso do BIM na etapa de operação estão relacionadas à falta de informação sobre os benefícios do BIM para esta etapa, a escassez de padronização dos dados necessários e à escassez de exemplos de uso a serem seguidos. Assim, dada a necessidade de pesquisas voltadas para a integração entre o BIM e a gestão das instalações, a O\&M de instalações habilitadas para o BIM se apresenta como um emergente campo de pesquisa (GAO; PISHDAD-BOZORGI, 2019).

Dentro deste contexto, esta pesquisa propõe investigar de forma quantitativa como têm sido desenvolvidas as pesquisas que tratam do BIM para a gestão da O\&M de instalações de edificações por meio de uma análise cientométrica da literatura científica. Neste sentido, acredita-se que este diagnóstico revele tópicos e tendências de pesquisa existentes bem como desafios e oportunidades para trabalhos futuros.

\section{METODOLOGIA}

A presente pesquisa utilizou o método cientométrico para investigar a literatura existente acerca dos usos BIM no campo da gestão das instalações. As análises cientométricas permitem ao pesquisador visualizar e mapear quantitativamente determinado domínio do conhecimento e, consequentemente, descobrir novas tendências de pesquisa bem como entender as estruturas de conhecimento já existentes (HE et al., 2017; WEN et al., 2021).

Para alcançar o objetivo desta pesquisa, inicialmente foram identificadas as principais publicações do campo estudado, as quais foram recuperadas da base de dados Web of Science (WoS). A escolha da WoS como repositório a ser adotado no presente trabalho se deve aos seguintes fatores: (1) a WoS é maior base de dados internacional (WEN et al., 2021); (2) a WoS apresenta uma melhor comunicação com aplicações de análise cientométrica como o VOSviewer e o CiteSpace, e; (3) pesquisa recentes que fizeram análises cientométricas no âmbito do campo de pesquisa do BIM utilizaram a WoS como base de dados principal (KHUDHAIR et al., 2021; LIU et al., 2021; WEN et al., 2021).

Para a pesquisa na base de dados WoS, a literatura foi recuperada através da associação entre as seguintes palavras-chave e operadores booleanos: ("building information modeling" OR "BIM") AND ("maintenance management" OR "facility management" OR "facilities management" OR "FM" OR "operation \& maintenance" OR "O\&M" OR "operations and maintenance" OR "operation" OR "maintenance" OR "BIM-enabled FM" OR "predictive maintenance" OR "post-occupancy" OR "refurbishment" OR "retrofit"). A busca foi definida como por "tópico", coletando assim as publicações que apresentavam as palavras-chaves investigadas em seus títulos, resumos, palavras-chave do autor ou keywords plus. O intervalo temporal da busca abrangeu todo o período desde o início de indexação de publicações na WoS, ou seja, de 1945 a 2021.

Como resultado da busca inicial, foram recuperadas 1548 publicações, as quais passaram por um processo de refinamento em três etapas, baseado nos seguintes critérios: (1) exclusão por tipo de documento - publicações de early access, material editorial, material editorial, resumo de reuniões, correções e publicações retratadas foram removidas, resultado em 1513 publicações; (2) exclusão por "Categorias do Web of Science" - 
categorias associadas às ciências da saúde foram deletadas, restando assim 1390 publicações, e; (3) exclusão por leitura dos títulos das pesquisas - trabalhos que não eram relacionados à modelagem da informação da construção foram deletados, o que levou à redução da coleção de artigos a um total de 1359 publicações, as quais foram adotadas para análise cientométrica após o processo de filtragem. Para conduzir a análise, as ferramentas CiteSpace e VOSviewer foram usadas neste estudo. O CiteSpace foi usado especificamente para conduzir análise de clusters. O VOSviewer, que conta o número de links e a forças total dos links para gerar uma rede de visualização (LAENGLE et al., 2018); foi usado para construir links para periódicos científicos, pesquisadores, países e palavras-chave baseado em coautoria, coocorrência ou cocitação.

\section{ANÁLISE DA BASE INTELECTUAL}

Este tópico apresenta uma análise quantitativa dos 1359 artigos selecionados. A análise cronológica irá permitir entender a distribuição temporal das pesquisas no campo, assim como as análises com base em autoria, país, organizações, palavras-chave e periódico irão permitir entender a evolução das pesquisas no campo de estudo. A priori, serão feitas análises com os resultados fornecidos pela própria WoS. Na sequência, serão feitas as análises com o CiteSpace e VOSviewer.

A Figura 1 apresenta a distribuição temporal das 1359 publicações. Percebe-se da Figura 1 que as publicações acerca do tema se iniciaram em 2003 e atingiram pico em 2019, quando 251 pesquisas foram publicadas. Ressalta-se ainda que se somados, os anos de 2017 a 2020 representam mais de 65\% das publicações. Da análise quantitativa das publicações, foi possível identificar ainda que as primeiras pesquisas foram publicadas em anais de eventos científicos (2003 - 2007). O trabalho de Vanlande, Nicolle e Cruz (2008) foi a primeira pesquisa de periódico a abordar conjuntamente o BIM e a gestão das instalações.

Figura 1 - Distribuição temporal das pesquisas

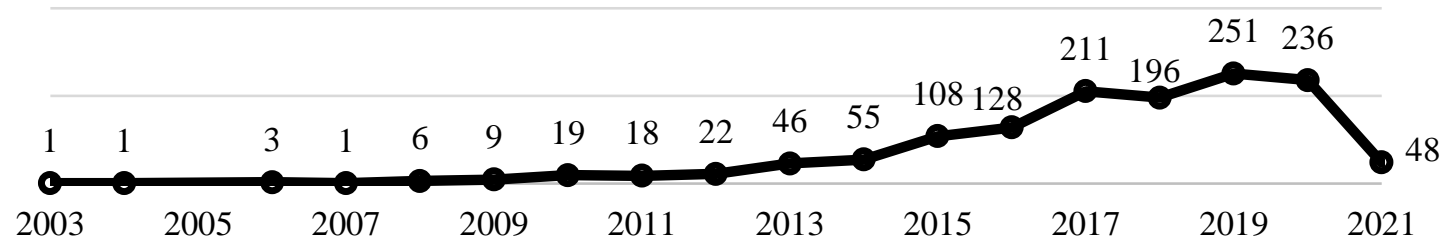

Fonte: Elaborado pelos autores.

Em relação ao tipo de documento, identificou-se que $54,82 \%$ das publicações são de artigos de pesquisa, 40,912\% são artigos de congresso e 5,224\% são artigos de revisão. No que diz respeitos às categorias do Web of Science, foi possível identificar que as três principais categorias às quais os artigos se enquadram são: engenharia civil; tecnologia de construção de edifícios e aplicações interdisciplinares de ciência da computação.

A coocorrência de palavras-chave dos autores foi investigada com auxílio do VOSviewer. As palavras-chave representam os principais conteúdos de documentos publicados e mostram as principais áreas de pesquisa dentro das fronteiras do conhecimento. Na rede de palavras-chave apresentada pelo VOSviewer, a distância indica a força da ligação entre dois domínios do conhecimento, quanto maior a distância, mais fraca a ligação entre dois itens. O tamanho do item indica o número de ocorrências e as cores representam os diferentes domínios do conhecimento agrupados pelo VOSviewer. Para criação do mapa, foi configurado no VOSviewer o número mínimo de 20 ocorrências da palavra-chave, de forma que 53 publicações atenderam a este limite. 
A Figura 2 mostra a rede de palavras-chave com 53 nós. É possível identificar pela análise da Figura 2 a existência de seis agrupamentos principais, a saber: (1) Cluster 1, representado na cor vermelha, com foco em gestão e construção; (2) Cluster 2, na cor verde, com foco na análise do ciclo de vida, eficiência energética e sustentabilidade; (3) Cluster 3, na cor azul escuro, centrado na interoperabilidade; (4) Cluster 4, na cor amarela, voltado para gestão de ativos; (5) Cluster 5, na cor lilás, com cerne nos frameworks, integração e conhecimento, e; (6) Cluster 6, em azul claro, tratando da realidade aumentada, entre outros aspectos. É importante notar também que os termos com maior ocorrência em cada cluster são termos genérico (Bim, Building Information Modeling, Management, Construction, Facility management, entre outros), que não trazem informações precisas acerca do que é de fato estudado em um dado cluster. Sendo assim, é fundamental analisar a força de ligação das demais palavras-chave que se ligam a estes termos que apresentam maior número de ocorrências. A análise da distribuição temporal da ocorrência das palavras chave, também permitida pelo VOSviewer permitiu entender que palavras-chave como infraestrutura, desempenho, otimização, Gêmeos Digitais, reforma e análise do ciclo de vida têm sido mais citadas nos últimos anos. Assim, estes campos se apresentam como os de maior potencial para desenvolvimento de pesquisas inovadoras e com maiores chances de apresentarem lacunas do conhecimento ainda a serem preenchidas.

Figura 2 - Mapa de coocorrência de palavras-chave

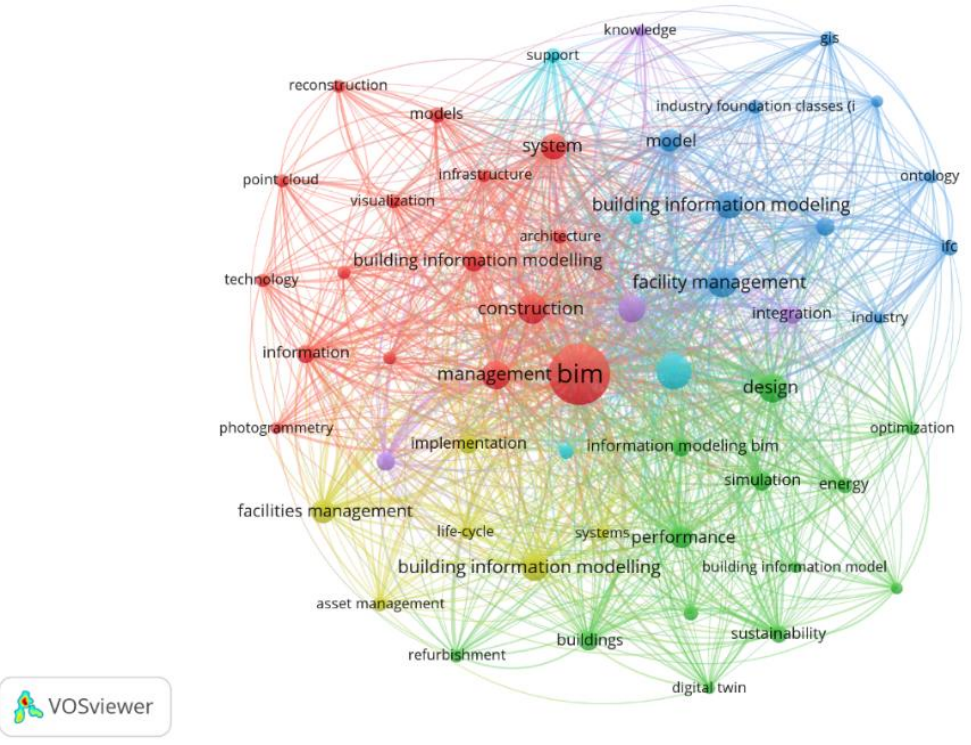

Fonte: Elaborado pelos autores.

No tocante às autorias, a WoS destaca Cheng JCP (16 publicações), Wang XY (16 publicações) e Wang $\mathbf{J}$ (12 publicações) como os três autores com maior quantidade de publicações no tema. Sendo assim, quando da revisão deste campo de pesquisa, estes são autores cujas pesquisas devem ser priorizadas. Cabe frisar que estes autores têm atuado em universidades da China, Australia e Coreia do Sul. A rede de colaboração científica foi delineada com auxílio do VOSviewer através do mapa de coautoria de autores apresentado na Figura 3. Para tal, foi configurado um número mínimo de cinco documentos por autor e 60 autores atenderam ao limite estabelecido distribuídos em 29 clusters. É possível inferir da Figura 3 o grande isolamento de autorias nas pesquisas, dado que dos 29 cluster, 14 são formados por apenas um autor e sete por dois autores. Além disso, através da Figura 3 é possível perceber ainda que os autores Cheng JCP, líder de publicações no campo, tende a trabalhar com forte colaboração com outros autores. 


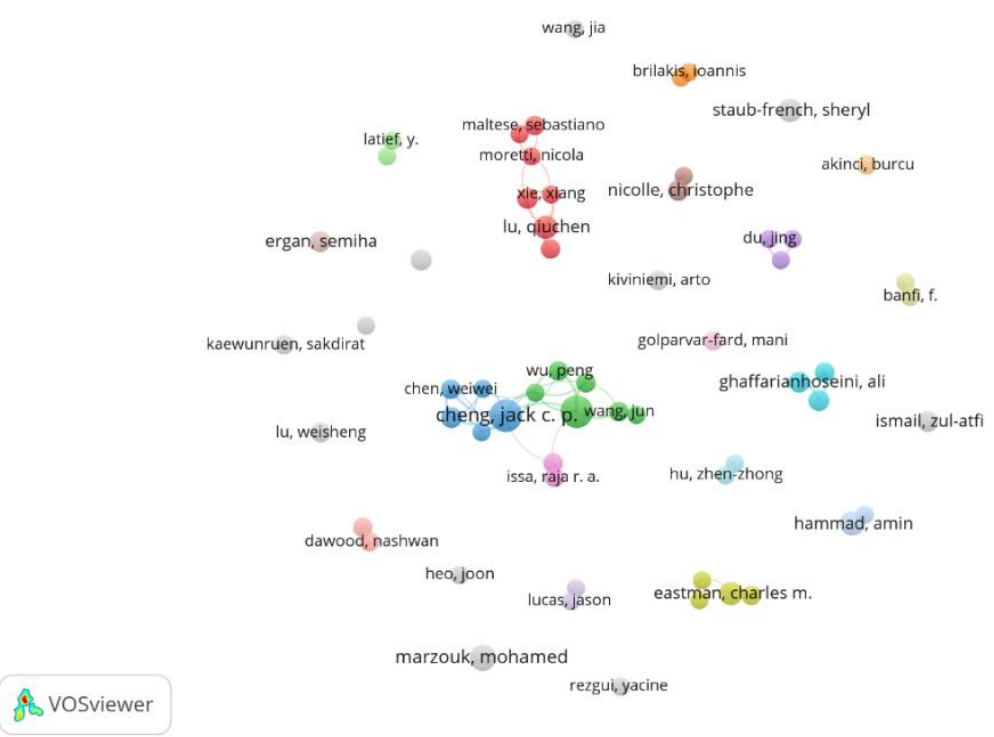

Fonte: Elaborado pelos autores.

Relativamente aos países, é possível perceber que a China (261 publicações), que também possui alguns dos autores com os maiores números de publicações; os Estados Unidos (208 publicações) e a Inglaterra (152 publicações) são os três maiores produtores de conhecimento neste campo de pesquisa. O Brasil, por sua vez, fica apenas entre os 23 países mais produtores de conhecimento com um total de 16 publicações. Em termos de cooperação, o mapa de coautoria de países é apresentado na Figura 4. Para tal, foi configurado um número mínimo de cinco documentos por país e 49 países atenderam ao limite estabelecido sendo distribuídos em 10 clusters. É possível notar da Figura 4, uma forte colaboração entre a China, Estados Unidos, Australia e Coreia do Sul. O Brasil tem colaborado mais fortemente com países como Espanha, Portugal, Estados Unidos e Inglaterra.

Figura 4 - Mapa de coautoria de países

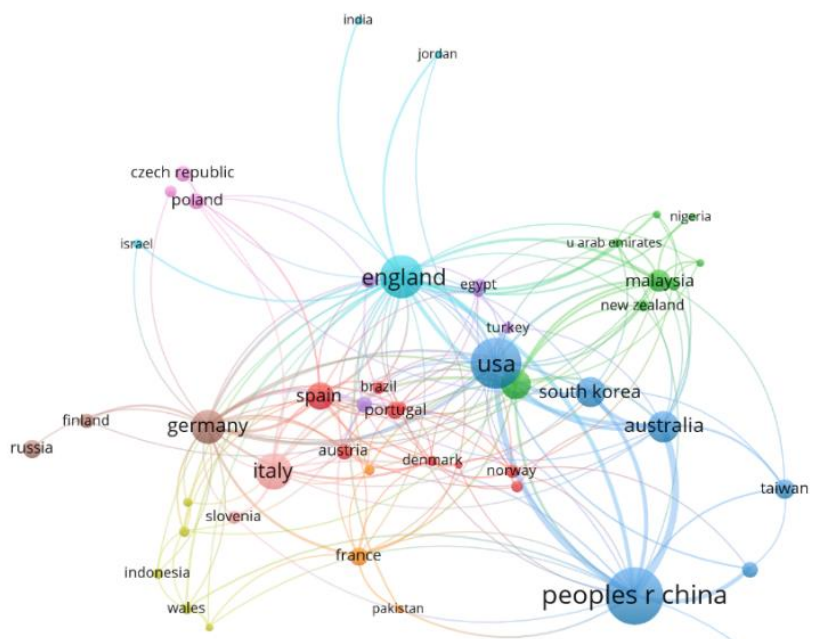

\& vosviewer

Fonte: Elaborado pelos autores. 
Em relação aos principais periódicos, destaca-se que os journals Automation in Construction, Procedia Engineering e Sustainability são os periódicos com maior número de publicações no campo e sozinhos somam mais de $16 \%$ do total de publicações. Com o uso do VOSviewer, a análise de cocitação de periódicos foi desenvolvida. A cocitação de periódicos ocorre quando duas publicações de dois diferentes periódicos citam a mesma publicação de outro periódico. A análise de cocitação permite identificar as literaturas mais importantes. O mapa de cocitação de periódicos é apresentado na Figura 5. Para um número mínimo de 100 citações, 40 periódicos foram incluídos. Conforme é característico dos mapas gerados pelo VOSviewer, o tamanho do círculo indica a relevância do item e as linhas indicam as relações de cocitação, já a quantidade e a espessura das linhas, por sua vez, indicam a força da ligação (link). A similaridade de coloração dos nós indica proximidade e relação entre os itens. Assim, percebe-se da Figura 5 que o periódico Automation in Construction possui grande influência e autoridade no campo de pesquisa abordado no presente trabalho.

Figura 5 - Mapa de cocitação de periódicos

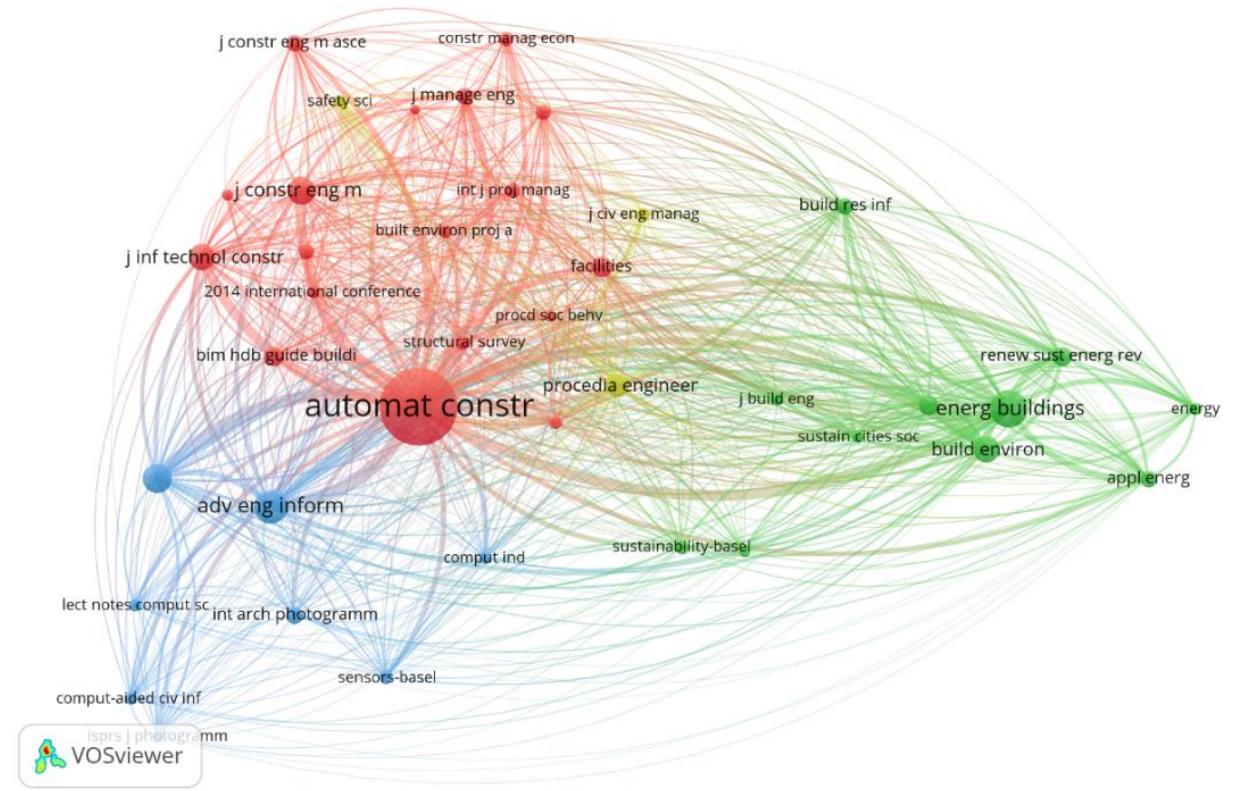

Fonte: Elaborado pelos autores.

A análise de agrupamento (clusters) foi desenvolvida com auxílio do CiteSpace e permite aos pesquisadores descriminar as frentes de pesquisa bem como explorar as correlações decorrentes. Os clusters de cocitação foram determinados com base na análise de cocitação. A Figura 6 apresenta o mapeamento de clusters. É possível verificar que a rede se mostrar bastante sobreposta e ramificada. Da análise, extrai-se ainda que 14 direções de pesquisa são delineadas, a saber: sistema de informação geográfica (SIG) (\#2), edificações escolares (\#9), gestão das instalações (\#0), edifícios verdes (\#3), funções da modelagem da informação da construção (\#1), ambientes virtuais (\#15), tecnologias de automação (\#4), suporte de automação (\#6), modelos de projeto (\#17), abordagens existentes (\#7), gestão do ciclo de vida da edificação (\#14, \#12, \#5), análise de modelagem de informações de construção de redes de utilidades internas (\#13). Além disso, é possível identificar que temas como SIG e edificações escolares têm sido abordados mais recentemente e também apresentam maior potencial de apresentarem lacunas do conhecimento ainda a serem preenchidas. Outrossim, do cruzamento dos dados das Figuras 2 e 6, pode-se perceber que os clusters gestão das instalações e gestão e construção foram encontradas em ambas as análises. 


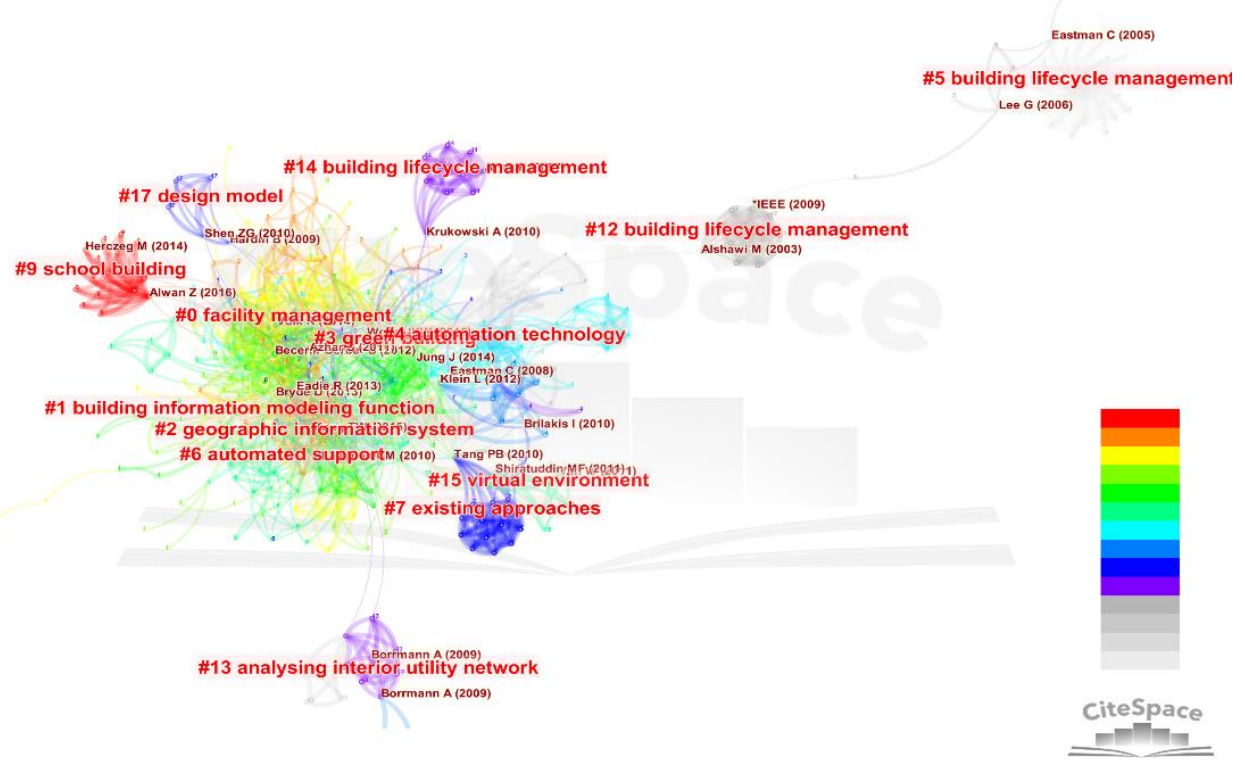

Fonte: Elaborado pelos autores.

\section{CONSIDERAÇÕES FINAIS}

A O\&M de instalações habilitadas para o BIM é um campo de pesquisa emergente, o que pode ser percebido através do crescimento da produção científica dentro do campo. Os últimos três anos chegam a representar mais de $65 \%$ das publicações. Neste sentido, a análise cientométrica proposta no presente trabalha é relevante por permitir um melhor delineamento do campo de pesquisa, facilitando a identificação dos mais relevantes periódicos, pesquisadores, pesquisas e tópicos de pesquisa, o que se mostra importante sobretudo para novos pesquisadores que atuam na área.

Em resumo, dentro do escopo da análise cientométrica desenvolvida na presente pesquisa, foi possível identificar que:

- A gestão da O\&M das instalações atrelada ao BIM tem potenciais lacunas do conhecimento associadas aos temas infraestrutura, desempenho, otimização, Gêmeos Digitais, reforma e análise do ciclo de vida, dado que estes temas têm sido os mais estudados nos últimos anos;

- Os autores Cheng JCP, Wang XY e Wang J possuem elevada expertise no campo, face ao elevado número de trabalhos publicados;

- A maior parte dos autores que possuem mais de cinco pesquisas acerca do tema tem trabalhado de forma isolada;

- As publicações da China, Estados Unidos e Inglaterra demonstram relevância dada a grande quantidade de publicações que estes países produzem;

- China, Estados Unidos, Australia e Coreia do Sul apresentam forte colaboração no desenvolvimento de pesquisas;

- O Brasil está entre os 23 países mais produtores de conhecimento com um total de 16 publicações e tem colaborado mais fortemente com Espanha, Portugal, Estados Unidos e Inglaterra;

- Os journals Automation in Construction, Procedia Engineering e Sustainability são os periódicos com maior número de publicações no campo.

No entanto, destacam-se algumas limitações do presente trabalho, como a adoção de uma única base de dados e a ausência de uma análise qualitativa dos artigos que compõem o grupo de dados avaliado, para além da análise das áreas de concentração e do título, tal qual foi feito nesta pesquisa; o que pode acabar incluindo temas que não são alvo do estudo. 
Assim, como sugestão de trabalhos futuros sugere-se: (1) investigação do tema em múltiplas bases de dados; (2) revisão da literatura dentro do campo, com a leitura e análise crítica das publicações de forma integral; (3) ampliação e aprofundamento das análises cientométrica desenvolvidas neste trabalho, e; (4) análise comparativa dos resultados através da aplicação de outras ferramentas de análise cientométrica.

\section{REFERENCIAS}

DONG, B.; O’NEILL, Z.; LI, Z. A BIM-enabled information infrastructure for building energy Fault Detection and Diagnostics. Automation in Construction, v. 44, p. 197211, 2014.

FAROOQ, J.; SHARMA, P.; SREERAMA KUMAR, R. Applications of building information modeling in electrical systems design. Journal of Engineering Science and Technology Review, v. 10, n. 6, p. 119-128, 2017.

FITZ, D. V.; SALEEB, N. Examining the quality and management of non-geometric building information modelling data at project hand-over. Architectural Engineering and Design Management, v. 15, n. 4, p. 297-310, 2019.

GAO, X.; PISHDAD-BOZORGI, P. BIM-enabled facilities operation and maintenance: A review. Advanced Engineering Informatics, v. 39, n. August 2018, p. 227-247, 2019.

HE, Q. et al. Mapping the managerial areas of Building Information Modeling (BIM) using scientometric analysis. International Journal of Project Management, v. 35, n. 4, p. 670-685, 2017.

KHUDHAIR, A. et al. Towards future BIM technology innovations: A bibliometric analysis of the literature. Applied Sciences (Switzerland), v. 11, n. 3, p. 1-21, 2021.

LAENGLE, S. et al. Thirty years of the International Journal of Computer Integrated Manufacturing: a bibliometric analysis. International Journal of Computer

Integrated Manufacturing, v. 31, n. 12, p. 1247-1268, 2018.

LI, J. et al. A review of currently applied building information modeling tools of constructions in China. Journal of Cleaner Production, v. 201, p. 358-368, 2018.

LIU, Z. et al. Blockchain and building information management (Bim) for sustainable building development within the context of smart cities. Sustainability (Switzerland), v. 13, n. 4, p. 1-17, 2021.

MULLER, M. F. et al. A systematic literature review of interoperability in the green Building Information Modeling lifecycle. Journal of Cleaner Production, v. 223, p. 397-412, 2019.

OKAKPU, A. et al. Exploring the environmental influence on BIM adoption for refurbishment project using structural equation modelling. Architectural Engineering and Design Management, v. 16, n. 1, p. 41-57, 2020.

VANLANDE, R.; NICOLLE, C.; CRUZ, C. IFC and building lifecycle management. Automation in Construction, v. 18, n. 1, p. 70-78, 2008.

WEN, Q. J. et al. The progress and trend of BIM research: A bibliometrics-based visualization analysis. Automation in Construction, v. 124, n. July 2020, p. 103558, 2021. 